\title{
Fourier Analysis of Ultrasonic TOFD Signals for Defect Detection in Austenitic Stainless Steel Welds
}

\author{
Angam Praveen \\ School of Electrical \\ and Electronics \\ Engineering \\ SASTRA \\ UNIVERSITY \\ Thanjavur, India
}

\author{
K Vijayarekha \\ School of Electrical \\ and Electronics \\ Engineering \\ SASTRA \\ UNIVERSITY \\ Thanjavur, India
}

\author{
Saju T Abraham \\ Quality Assurance \\ Division \\ Indira Gandhi Centre \\ for Atomic Research \\ Kalpakkam \\ India
}

\author{
B Venkatraman \\ Radiological Safety \\ Group \\ Indira Gandhi Centre \\ for Atomic Research \\ Kalpakkam \\ India
}

\begin{abstract}
Non Destructive testing of structural materials is an important activity in many industries. Ultrasonic NDT is one such method widely used in this field. Pulse Echo and Through Transmission are conventional testing techniques which use reflected and transmitted sound energy respectively. But these techniques are highly dependent on defect orientation. Time of Flight Diffraction (TOFD) is a recent method which uses diffracted sound energy to detect the defects and is independent of orientation of the defects in the material. TOFD signals are dominated by noise arriving from various sources namely instrumental noise, material (structural) noise, thermal noise etc. Signal processing is an important step in the evaluation of the signals for detecting, positioning and characterizing the defects. Fourier Transform is widely used in the analysis and de-noising of signals of different kind. In this paper Ultrasonic TOFD signals obtained from Austenitic Stainless Steel weldments are analyzed using Fourier Transforms.
\end{abstract}

\section{General Terms}

NDT, Ultrasonic Testing, TOFD, Signal Processing, Fourier analysis.

\section{Keywords}

Ultrasonic, Defect, NDT, TOFD, Fourier Transform, Austenitic Stainless Steel, Welds.

\section{INTRODUCTION}

Evaluation of materials in a non destructive manner is an important requirement in many strategic industries. Ultrasonic Testing (UT) is very popular among the available NDT techniques due to its simple principle, ease in analysis and cost effectiveness. Some of the conventional techniques in UT include Pulse Echo and Through Transmission technique. While pulse echo technique uses the sound energy reflected from the defects, through transmission technique uses reduction in transmitted energy due to the presence of defect. These techniques are not reliable in determining the exact location of the defects due to the dependence on orientation of the reflecting surface. These techniques in turn do not provide the information about the size of the defect accurately. These limitations led to the invention of a new technique namely Time of Flight Diffraction (TOFD) technique [1-7] which is independent of the orientation of the defects.

Ultrasonic inspection of weldments is of mandatory in any process industry. As the ultrasonic waves propagate through the weld material, it interacts with the grains which results in scattering of the waves. This becomes more severe when the material is an austenitic stainless steel weld. Austenitic stainless steel is the major material used in nuclear industry because of its superior mechanical properties under high temperature and radiation environments. Codes and standards recommend ultrasonic inspection of these welds with stringent quality requirements. The austenitic stainless steel welds are highly anisotropic in nature due to the columnar grain structure. Scattered ultrasonic waves from the columnar grains introduce noise in the received signals which is spread throughout the bandwidth with different intensity. These noises may mask the defects. Proper analysis of signals is important for the detection, positioning and characterization of the defects accurately. Fourier transform is a conventionally available tool for the frequency analysis and smoothening of the signal. In this paper FFT is used for detecting the presence of defects, analysis and de-noising of ultrasonic TOFD signals obtained from Austenitic Stainless Steel welds.

\section{TIME OF FLIGHT DIFFRACTION}

TOFD is a recent technique in ultrasonic non destructive testing of materials [8]. It uses the diffraction principle for testing. Fig. 1 shows the principle of TOFD. When ultrasonic waves propagating through the material encounters a sharp edge or tip they get diffracted and results in small oscillatory waves of finite duration. Since diffracted waves are obtained from the tips of the defect they are independent of the defect orientation. The diffracted waves from the upper and lower tip of the defect are used in sizing of the defect.

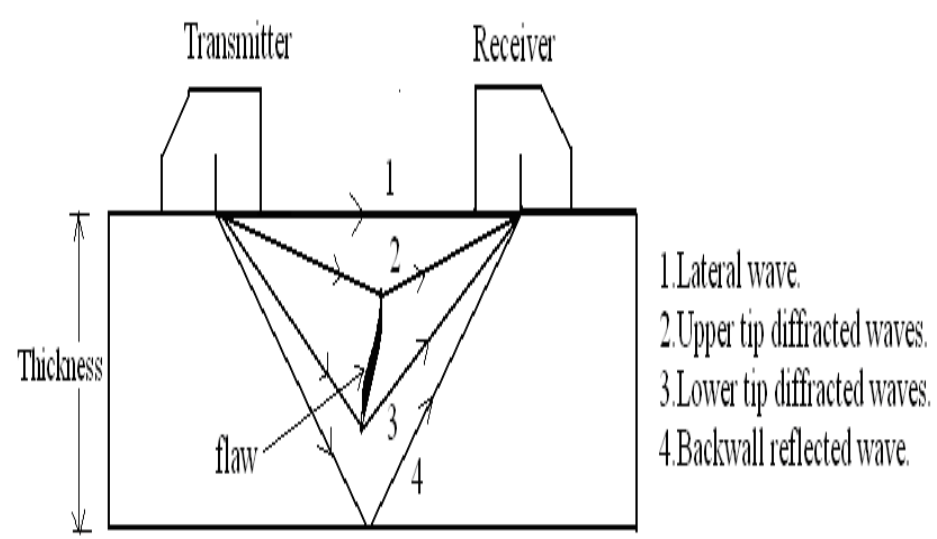

Fig 1: TOFD Schematic set-up 


\section{EXPERIMENTAL DETAILS}

A $25 \mathrm{~mm}$ thick weld pad of austenitic stainless steel grade 304L has been fabricated by Shielded Metal Arc Welding method. A slag inclusion of length $40 \mathrm{~mm}$ was intentionally introduced in the weld. This weld pad has been scanned by TOFD technique. A $4 \mathrm{MHz}, 45^{\circ}$ longitudinal angular probe was used to launch waves into the material. The experiment was conducted at room temperature. The equipment used was MICROPLUS from AEA Technology, UK. The channel gain was set to $80 \mathrm{~dB}$. TOFD A-scan signals were acquired using a digital storage oscilloscope (YOKOGAWA -DL9140, 5GS/s, $1 \mathrm{GHz})$. Fig. 2 shows the experimental set-up. Signals were acquired from defective as well as non-defective areas and two particular signals are shown in Fig. 3 and Fig. 4.

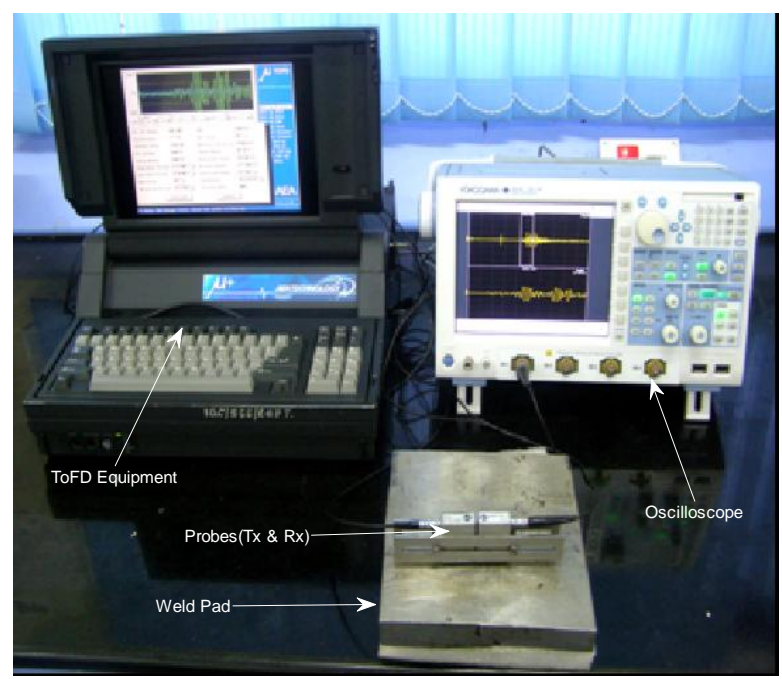

Fig 2: TOFD Experimental set-up

\section{FOURIER ANALYSIS}

Fourier series representation gives the information of frequency components present in a given time varying signal. This is best suitable when the signal is periodic [9]. Extension of Fourier series is Fourier Transform which can be used to represent non periodic signals. FFT being the algorithm to implement DFT is very useful in the spectral analysis of large group of discrete time domain signals [10-11]. Ultrasonic Testing involves non stationary signals i.e. the frequency components present in the signal vary widely over time.

Fig. 5 shows the Fourier spectrum of the signals given in Fig. $3 \& 4$. It can be observed that the presence of defect in the material is indicated by sharp peaks near the dominant frequency component region [12]. This method can be used for understanding the presence of defects in a material. By this method, one can easily determine the presence of defects in a material by looking at the large peaks unlike the smooth variation in case of flawless signal. To evaluate this method, several weld pads with different types of defects have been studied. This method has been applied to these weld pads and some of the results are shown here. Fig. $6,7 \& 8$ shows results for defects of type lack of penetration, lack of fusion and slag respectively.

Spectral analysis using Fourier transform gives information about the presence of defects but no information about the time of occurrence of defect signals. To estimate the location of the defects in the material, the A-scan signal has to be evaluated. The time varying A-scan signals from the austenitic stainless steel is highly influenced by the structural noise. Fourier transform can be applied to de-noise the signal. Fig. 9 shows a de-noised signal and the corresponding original signal obtained from an austenitic stainless steel weld. This has been achieved by simple thresholding method. The denoised signal enables proper positioning of the defect from the surface of the material.

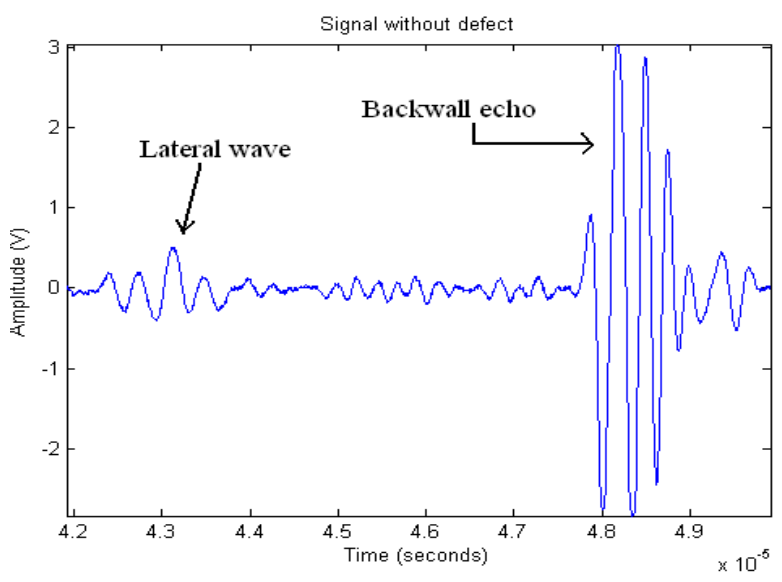

Fig 3: A-scan Signal Without Defect

Presence of small defect such as the one shown in Fig. 10 may not be confirmed by Fourier analysis due to its coinciding spectrum with that of the defectless spectral region. Fig. 11 shows the spectrum of the signal with and without defect for a small size defect. Thus it is observed that, in the case of austenitic stainless steel welds, Fourier analysis can be used for detection of defects with significant size only

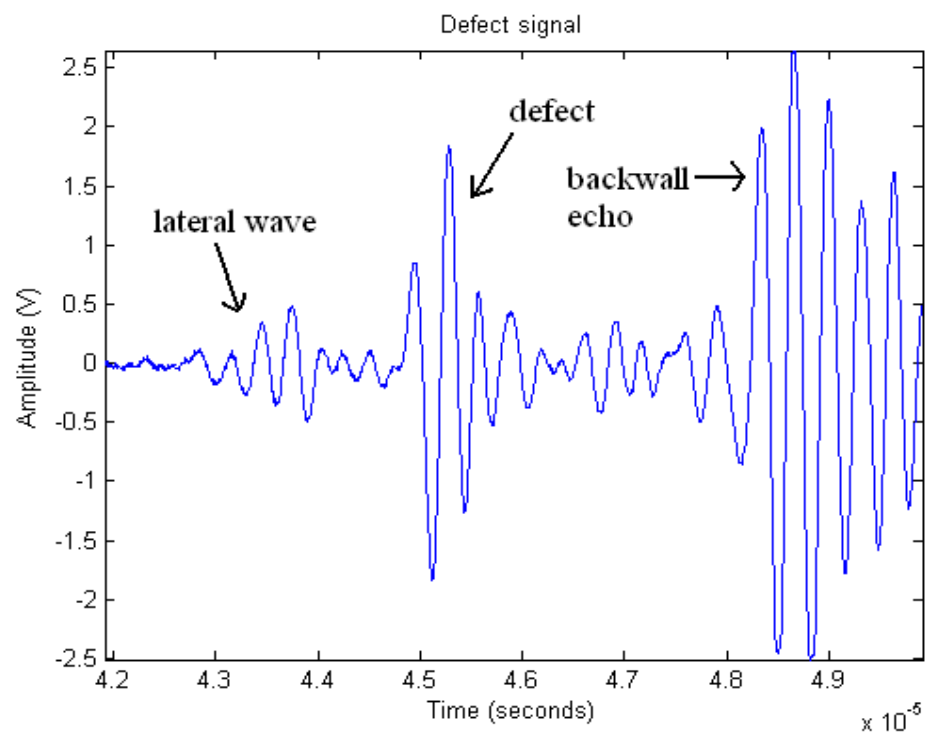

Fig 4: Signal With Defect 


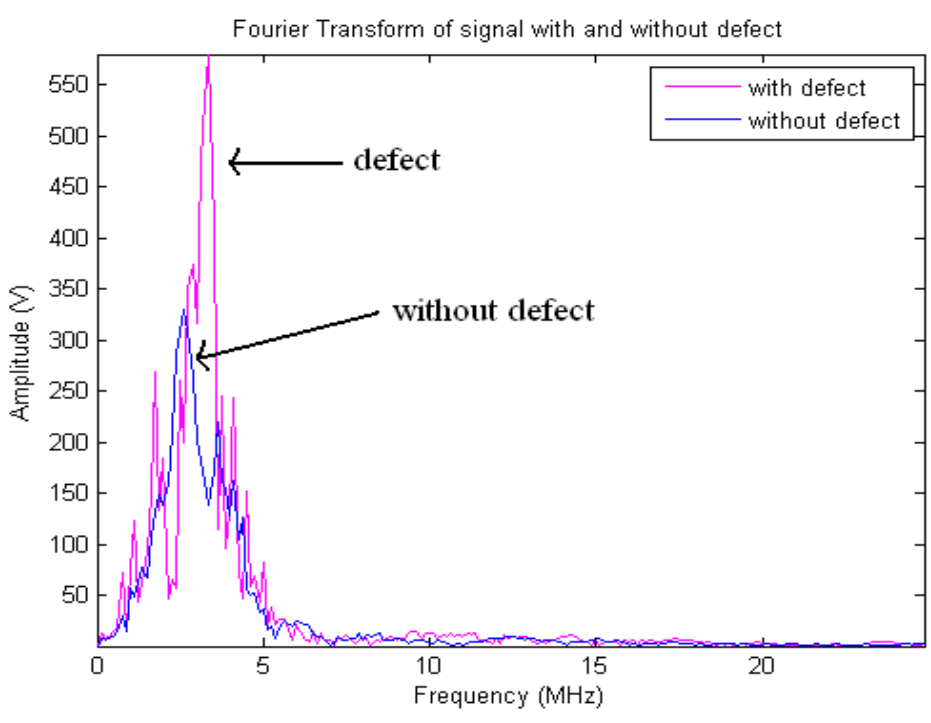

Fig 5: Spectrum with and without defect

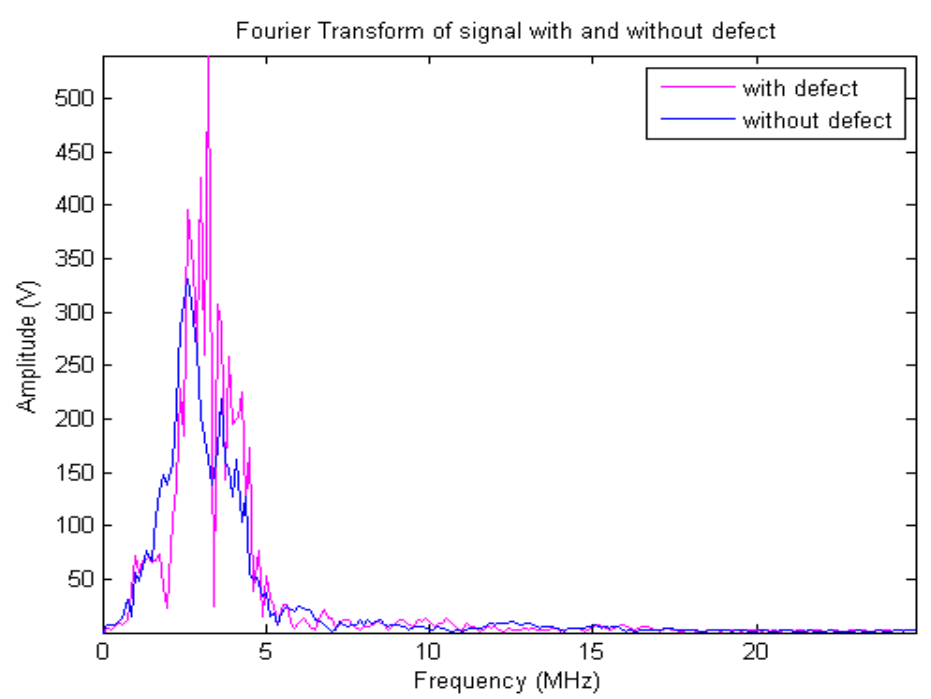

Fig 6: Spectral comparison for a lack of penetration defect type

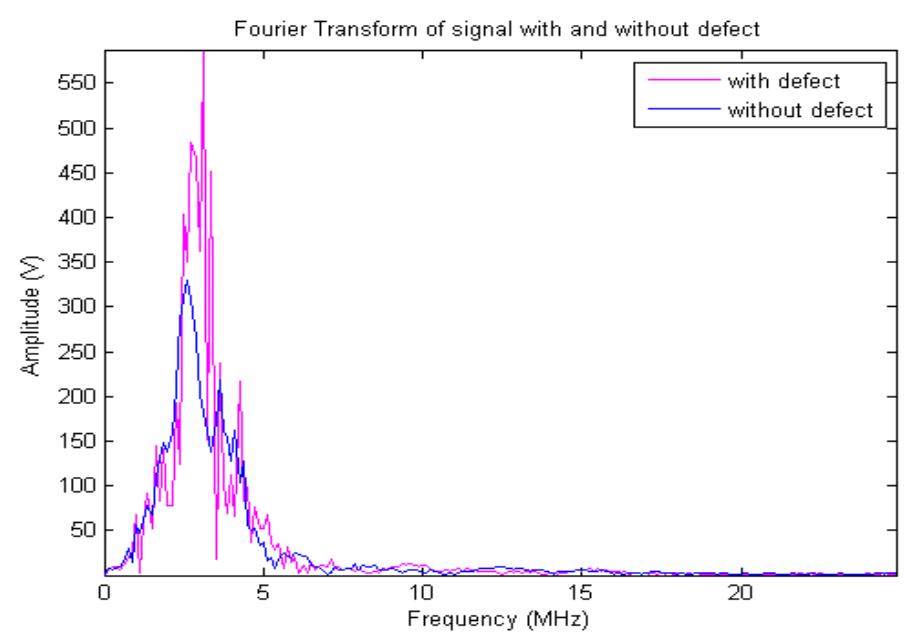

Fig 7: Spectral comparison for lack of fusion defect type

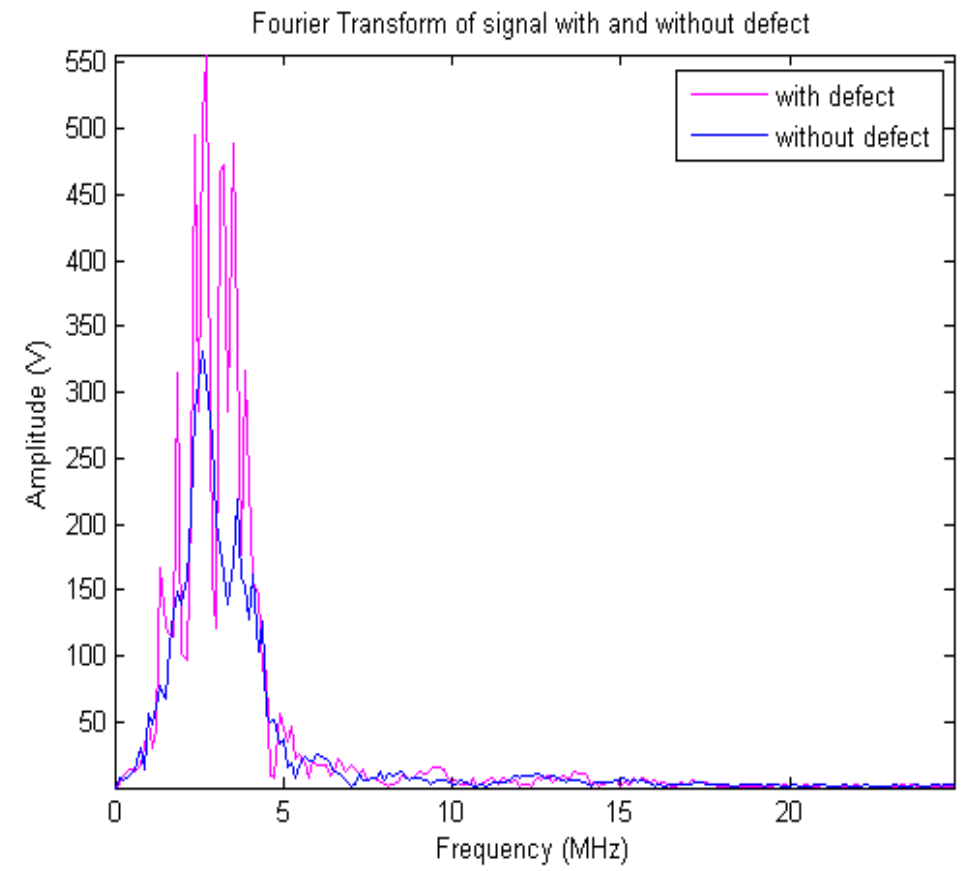

Fig 8: Spectral comparison of slag defect type

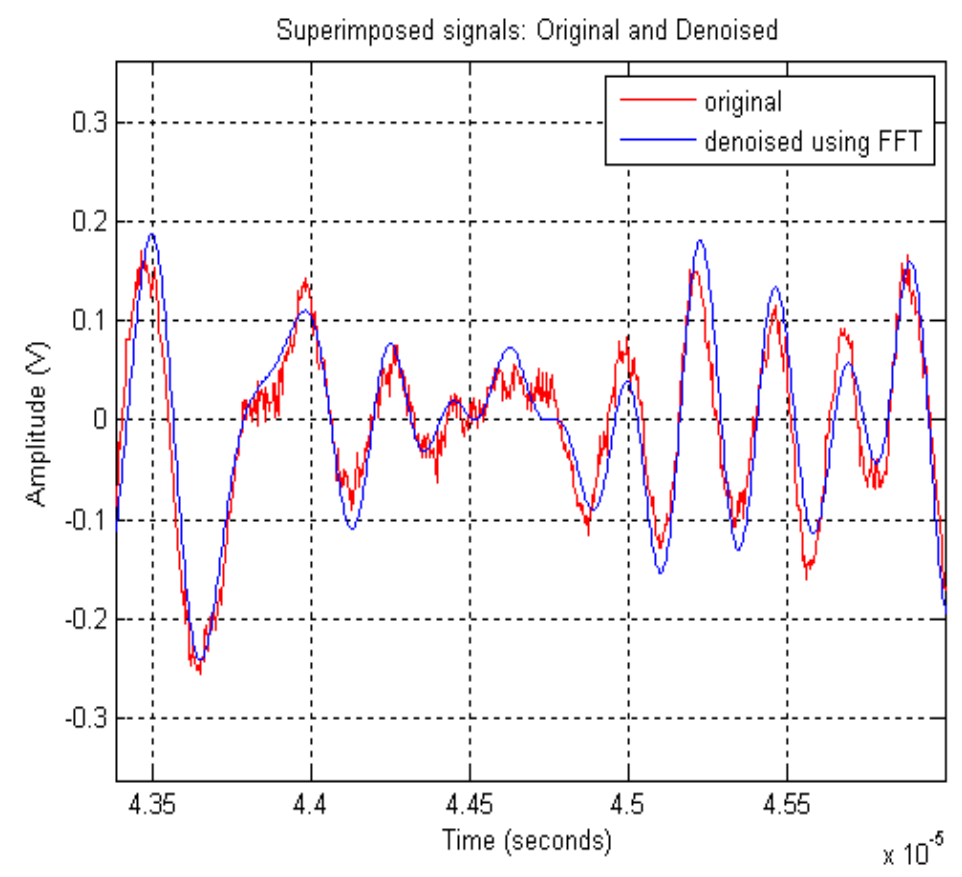

Fig 9: Original and de-noised signal 


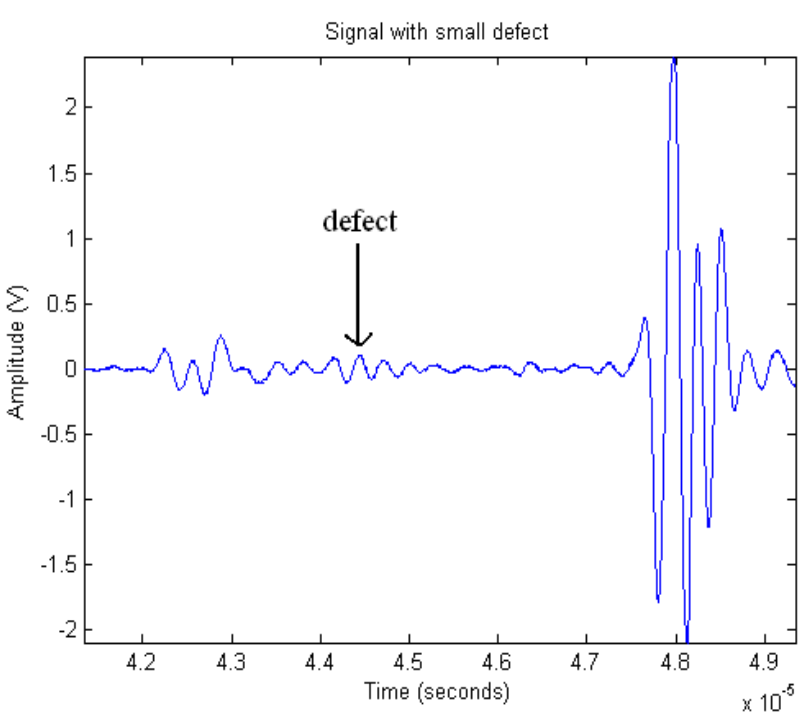

Fig 10: TOFD signal showing small Defect

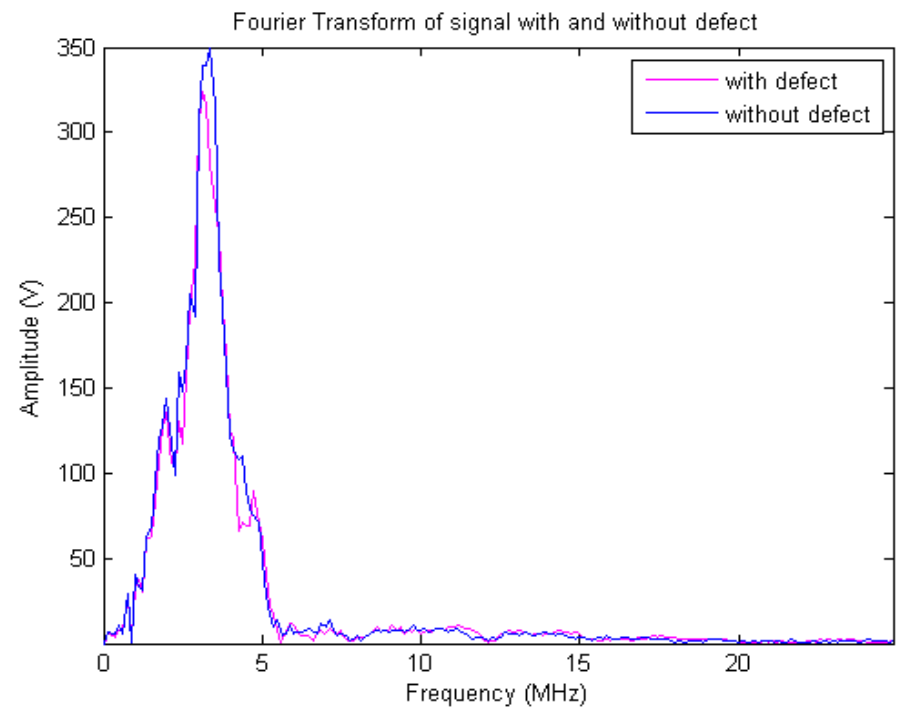

Fig 11: Spectrum of signal with and without small defect

\section{CONLUSION}

Ultrasonic Testing is one of the widely non-destructive testing methods in the evaluation of structural components especially in nuclear reactors. Conventional ultrasonic techniques fail in defect detection due to the random orientation of defects in many cases. TOFD based techniques provide accurate detection, sizing and characterization of defects. In this paper TOFD signals have been used for the evaluation of austenitic stainless steel welds. DSP tools play an important role in enhancing the defect detection in the presence of noise in the acquired signals. Fast Fourier Transform (FFT) has been used in this paper to determine the presence of defects and also to de-noise the signals for enhanced defect detection. A thresholding method in FFT has been applied to de-noise the signals from the austenitic stainless steel welds. It has been observed that it has greater ability to detect defects with significant size. FFT is also capable in de-noising of noisy TOFD signals for enhanced defect visibility.

\section{REFERENCES}

[1] Temple J.A.G., :Time-of-flight inspection: theory", Nulcear Energy, 1983,22, No. 5, Oct.,pp 335-348.

[2] Ogilvy J.A. and Temple J.A.G.,"Diffraction of elastic waves by cracks:application of time of flight inspection", ultrasonics, Nov. 1983, pp 259-268.

[3] Verkooijen J., "TOFD used to replace radiography", Insight Vol.37 No.6 June 1995, pp 433-435.

[4] Silk M.G., "An evaluation of the performance of the TOFD techniques as a means of sizing flaws, with particular reference to flaws with surved profiles", Insight vol.38, No.4, April,1996.

[5] Sony Baby, Balasubramaniam T., Pardikar R.J., Palaniyappan M. and Subbaratnam R., "Time of flight diffraction(TOFD) technique for accurate sizing if cracks embedded in sub-cladding",Insight vol.45, No.9, September, 2003

[6] M. Riahi and M. R. Abolhasany, "Substitution of the Time-of-Flight Diffraction Technique for Nondestructive Testing of Welds and Thick Layers of Steel: A Comparative Investigation", Russian Journal of Nondestructive Testing, Tehran, Iran Vol. 42, No. 12, 794-801

[7] Tianlu Chen, Peiwen Que, Oi Zhang, and Qingkun Liu, "Ultrasonic Nondestructive Testing Accurate Sizingand Locating Technique Based on Time-of-Flight-Diffraction Method", Russian Journal of Nondestructive Testing, 2005, Shanghai, China, Vol. 41, No. 9, 57-68, 594-601.

[8] Charles Worth J.P., Temple J.A.G., "Engineering application of ultrasonic time of flight diffraction", $2^{\text {nd }}$ ed. Research Studies press Ltd. 2001.

[9] S.K. Mitra, "Digital Signal Processing: A Computer Based Approach" $2^{\text {nd }}$ ed. New York: McGraw-Hill, 2001.

[10] Shalil Mohammed Shalil, "Fourier Transform-Signal Processing", April 2012.

[11] P.Munshi, "Fourier vision in NDT",Proc. Fourteenth World Conference on Nondestructive Testing(1996), Oxford IBH, New Delhi, pp1817-1820.

[12] Po-Liang Yeh, Pei-Ling Liu,'Application of the Wavelet transform and enhanced Fourier spectrum in Impact echo test', NDT\&E International, 2008, Vol. 41, 382-394. 